\title{
Length of stay overestimates severity of post-ERCP pancreatitis: Is it time to revise the consensus definition?
}

\section{다)(1) $\odot$}

Authors

Mahya Faghih ${ }^{1}$, Amitasha Sinha ${ }^{1}$, Robert A. Moran ${ }^{1}$, Elham Afghani ${ }^{1}$, Yuval A. Patel ${ }^{1}$, Andrew C. Storm ${ }^{1}$, Ayesha Kamal', Venkata S. Akshintala ${ }^{1}$, Atif Zaheer ${ }^{3}$, Anthony N. Kalloo' ${ }^{1,2}$, Vivek Kumbhari ${ }^{1}$, Mouen A. Khashab ${ }^{1}$, Vikesh K. Singh ${ }^{1,2}$

\section{Institutions}

1 Division of Gastroenterology, Johns Hopkins Medical Institutions, Baltimore, Maryland, United States

2 Pancreatitis Center, Johns Hopkins Medical Institutions, Baltimore, Maryland, United States

3 Department of Radiology and Radiological Science, Johns Hopkins Medical Institutions, Baltimore, Maryland, United States

submitted 12.12 .2017

accepted after revision 25.4.2018

Bibliography

DOI https://doi.org/10.1055/a-0624-2491 |

Endoscopy International Open 2018; 06: E838-E843

(c) Georg Thieme Verlag KG Stuttgart · New York

ISSN 2364-3722

\section{Corresponding author}

Vikesh K. Singh, MD, MSc, Johns Hopkins Hospital, Division of Gastroenterology, 1830 E. Monument Street, Room 428, Baltimore, MD 21205

Fax: +1-410-614-7631

vsingh1@jhmi.edu
ABSTRACT

Introduction Length of stay (LOS) is an important determinant of the severity of post-ERCP pancreatitis (PEP) in the consensus definition. The aim of our study was to evaluate and compare severity of PEP based on the revised Atlanta classification (RAC) and the consensus definition.

Patients and methods Between 1/2000 and 12/2011, all adult patients admitted with suspicion of PEP after outpatient ERCP were evaluated. PEP was defined using the RAC, but the severity of PEP was defined using both revised Atlanta and consensus definitions.

Results A total of 341 patients (mean age 49 years and $75 \%$ females) were diagnosed with PEP. The consensus definition classified $57 \%, 37 \%$, and $8 \%$ of patients with mild, moderate, and severe PEP, respectively. The RAC diagnosed $94 \%$, $6 \%$, and $0 \%$ with mild, moderate, and severe acute pancreatitis, respectively. Of the patients diagnosed with moderatesevere PEP by consensus definition, only $12.5 \%$ had clinical parameters of pancreatitis severity, such as acute fluid collection(s), pancreatic necrosis, transient organ failure and/ or required percutaneous or surgical drainage, while $87.5 \%$ were classified only based on a LOS $\geq 4$ days. The most common reason for increased LOS was persistent post-procedural abdominal pain in $47 \%$ of patients, followed by other reasons not related to pancreatitis in $17 \%$.

Conclusion The consensus definition overestimates the rates of severe PEP when compared to the RAC. The majority of PEP patients classified as moderate-severe PEP have extended LOS, due to post-procedural abdominal pain rather than complications of PEP.

\section{Introduction}

Acute pancreatitis (AP) is the most common complication of endoscopic retrograde cholangiopancreatography (ERCP) with an incidence of $2 \%$ to $16 \%[1,2]$. The consensus definition of post-ERCP pancreatitis (PEP) has been most commonly used for diagnosing and determining the severity of PEP [3]. The consensus definition has also been used in $75 \%$ of randomized controlled trials (RCT) of PEP prophylaxis [4]. However, shortly after the consensus definition for PEP was published, the Atlanta classification of 1992 proposed different criteria for the diagnosis and classification of severity in AP [5]. The revised Atlanta classification (RAC) of 2012 incorporated fundamental advances in our understanding of severity in AP and has subsequently been adopted as the primary severity classification for clinical studies in AP [6].

According to the consensus definition, mild and moderate PEP are defined by the length of hospital stay (LOS), with less 
than 3 days being mild and 4 to 10 days being moderate. Severe PEP is defined as a LOS $>10$ days and/or the presence of local complications (acute fluid collections, pancreatic necrosis and pseudocyst), need for intervention or death. Mild AP according to the RAC is a self-limited illness with no organ failure and/or local complications which corresponds to not only mild but also moderate PEP according to the consensus definition. Presence of local complications, transient ( $<48$ hours) organ failure (TOF) and/or exacerbation of comorbid diseases is defined as moderate AP according to the RAC. Local complications are associated with significant morbidity but are not the primary determinant of mortality in AP and, therefore, are not incorporated into the RAC definition of severe disease. The RAC defines severe AP only as persistent (>48 hours) organ failure (POF), which is the primary determinant of mortality in AP [7]. The consensus definition has undergone no modification since its formulation and, as a result, fails to incorporate POF and continues to rely on LOS, a highly subjective parameter, for defining severe PEP [8].

The primary aim of this study was to compare the severity of PEP as defined by the consensus definition and the RAC. The secondary aim of this study was to determine and compare PEP and non-PEP related factors in those patients who had an extended LOS.

\section{Patients and methods}

\section{Patient population}

We conducted a retrospective cohort study of 4,299 outpatients who underwent ERCP as a primary procedure and were subsequently admitted for suspicion of post-ERCP pancreatitis (PEP) between January 1, 2000 and December 31, 2011. We did not include patients from 2012 and onwards due to the fact that the RAC was disseminated to clinicians in 2012, prior to its publication in early 2013 [6]. Knowledge of the RAC could have potentially impacted the severity classification of a patient with PEP using the consensus definition if, for example, a clinician discharged a PEP patient with resolved organ failure before 4 days of hospitalization. In our clinical practice, all patients with persistent, new-onset post-procedural abdominal pain or intensification of their baseline abdominal pain after ERCP are admitted for observation. These patients were identified using the International Classification of Diseases, Ninth Revision, Clinical Modification (ICD-9-CM) diagnosis and procedure codes (CPT) for AP and ERCP which were then ascertained through chart review. Demographic, clinical and procedural data were collected for all patients by reviewing paper and electronic medical records.

Exclusion criteria included: 1) complications of ERCP other than PEP (perforation, post-sphincterotomy bleeding and cholangitis); 2) a prior episode of AP within 2 months of ERCP; 3 ) repeat admissions for PEP other than the index admission; 4) admission after an ERCP for an indication other than suspected PEP; 5 ) patients $<18$ years of age; and 6 ) patients with missing or incomplete records ( $>$ Fig. 1).
2929 admissions with an ICD9-CM diagnosis of acute pancreatitis (AP) and ERCP between 1/2000 - 12/2011

$406(13.9 \%)$ were excluded due to:

- $123(4.2 \%)<18$ years

- 283 (9.7\%) no ERCP on records

2523 adult admissions with a diagnosis of AP and an ERCP

1228 (41.6\%) were excluded due to:

- 97 (3.3\%) AP episode within 2 months

- $72(2.5 \%)$ perforation

- 57 (1.9\%) bleeding

- 350 (11.7\%) in-patient ERCP

- 292 (9.9\%) non-ERCP related admission

- 360 (12.3\%) repeat admissions

1295 adult admissions with suspected diagnosis of post-ERCP pancreatitis

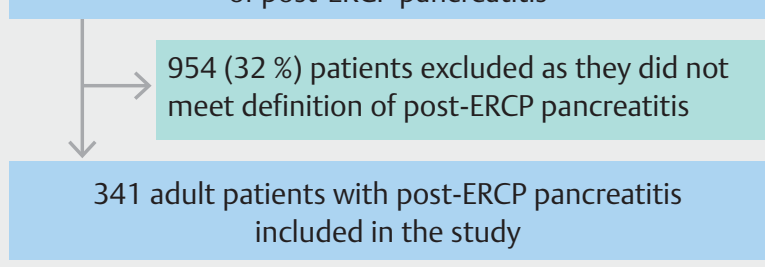

- Fig. 1 Flow diagram for inclusion and exclusion in study cohort.

\section{Definitions}

PEP was defined using the RAC [6]. The severity of PEP was categorized into mild, moderate and severe using both the RAC [6] and the consensus criteria [3]. The RAC defines AP as presence $\geq 2$ of the following: characteristic acute abdominal pain post-ERCP, serum amylase and/or lipase $\geq 3$ times the upper limit of normal after 24 hours of ERCP; and/or an abdominal computed tomography (CT) scan with findings consistent with AP [6]. The RAC defines mild AP as absence of organ failure and/ or local or systemic complications (acute peripancreatic fluid collection(s), pancreatic pseudocyst(s), acute necrotic collection(s), and walled-off necrosis), moderate AP as presence of TOF and/or local/systemic complications and severe AP as presence of POF [6]. The consensus criteria define mild PEP as an unplanned admission/length of stay (LOS) of 2 to 3 days, moderate PEP as an admission of 4 to 10 days, and severe PEP as an admission > 10 days and/or presence of any of the following: pseudocyst(s), pancreatic necrosis, surgical or percutaneous intervention, or mortality [3]. Acute fluid collection(s) and pancreatic necrosis were defined according to the RAC. Persistent (> 48 hours) organ failure was defined as a Marshall score $\geq 2$ in the renal, pulmonary and/or cardiovascular system(s). Organ failure was assessed each day during the entire period of hospitalization, based on the most extreme laboratory value or clinical measurement within any 24-hour period. Death was defined as mortality occurring during hospitalization. Other clinical 
- Table 1 Demographics, clinical characteristics and outcomes of the study cohort.

\begin{tabular}{|c|c|}
\hline & $\mathrm{n}=\mathbf{3 4 1}$ \\
\hline \multicolumn{2}{|l|}{ Demographics } \\
\hline Age (years), mean $\pm S D$ & $48.9 \pm 14.6$ \\
\hline Female & $255(74.8)$ \\
\hline White & $261(76.5)$ \\
\hline \multicolumn{2}{|l|}{ Indication for ERCP } \\
\hline Suspected sphincter of Oddi dysfunction & $100(29.3)$ \\
\hline History of acute recurrent idiopathic pancreatitis & $86(25.2)$ \\
\hline Choledocholithiasis & $45(13.2)$ \\
\hline Chronic Pancreatitis & $42(12.3)$ \\
\hline \multicolumn{2}{|l|}{ Biliary stricture } \\
\hline - Benign & $16(4.7)$ \\
\hline - Malignant & $34(9.9)$ \\
\hline Stent removal & $7(2.1)$ \\
\hline Cholangitis & $9(2.6)$ \\
\hline Bile leak & $2(0.6)$ \\
\hline History of prior post-ERCP pancreatitis & $32(10.4)$ \\
\hline \multicolumn{2}{|l|}{ Outcomes } \\
\hline Acute fluid collection(s) & $12(3.5)$ \\
\hline Necrosis & $3(0.9)$ \\
\hline Percutaneous/surgical drainage & $2(0.6)$ \\
\hline \multicolumn{2}{|l|}{ Organ failure } \\
\hline - Transient & $6(1.8)$ \\
\hline - Persistent & 0 \\
\hline Mean LOS & $3.8 \pm 3$ \\
\hline Death & 0 \\
\hline $\begin{array}{l}\text { Values are expresses as number (\%), unless specified oth } \\
\text { deviation; ERCP, endoscopic retrograde cholangiopancr } \\
\text { length of stay }\end{array}$ & $\begin{array}{l}\text { e. SD, standard } \\
\text { raphy; LOS, }\end{array}$ \\
\hline
\end{tabular}

parameters leading to a LOS $\geq 4$ days included persistent postprocedure abdominal pain, persistent nausea/vomiting and intolerance of oral intake, ileus, fever, and elevated liver enzyme (s) and electrolyte abnormalities among patients with no markers of severe AP.

\section{Statistical analysis}

Demographic and clinical characteristics at time of admission were reported using descriptive statistics, as proportions (percentages) for categorical variables and means (standard deviation) for continuous variables. The difference between the categorical variables was calculated using the Pearson's chisquared test. A $P$ value $<0.05$ was deemed significant. All statis-

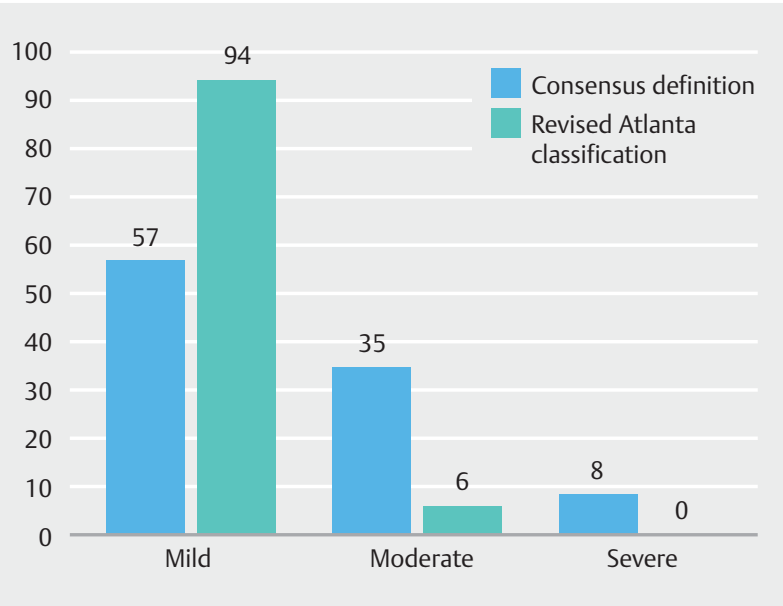

- Fig. 2 Frequency of mild, moderate and severe Post-ERCP pancreatitis according to the consensus definition and revised Atlanta classification (RAC) across 341 patients.

tical analysis was conducted using Stata version 13 (StataCorp LP, College Station, TX).

\section{Results}

- Fig. 1 displays the flow diagram of patients included in the final analysis. A total of 2,929 admissions (among 2262 patients) were noted with an ICD9-CM diagnosis of AP and an ERCP as a primary procedure over 12 years. Among these, 341 adult patients met the inclusion criteria and were included in the final analysis.

Demographic and clinical characteristics of the study cohort are described in $>$ Table 1 . The mean age was $48.9 \pm 14.6$ years, and $74.8 \%$ of the patients were female. The two most common indications for ERCP in this patient cohort were suspected sphincter of Oddi dysfunction (29.3\%) and acute recurrent idiopathic pancreatitis (25.5\%). A total of 172 patients (50.4\%) underwent abdominal imaging ( $\mathrm{CT}$ and or magnetic resonance imaging) during hospitalization. A total of 197 patients (57.8\%) had a LOS < 4 days (mean LOS $1.9 \pm 0.8$ days), 132 (38.7\%) between 4 to 10 days (mean LOS $5.8 \pm 1.7$ days) and 12 patients $(3.5 \%$ ) had an LOS $>10$ days (mean LOS $14.6 \pm 2.7$ days). Of those that had a LOS between 4 and 10 days, 105 patients (80\%) underwent imaging, while 11 patients (92\%) with a LOS > 10 days underwent imaging. There was no difference in LOS based on gender or indication for ERCP.

- Fig. 2 shows the distribution of patients with mild, moderate and severe PEP based on the consensus and RAC definitions. While the consensus definition diagnosed 194 patients (57\%) with mild, 121 patients (35\%) with moderate and 26 patients (8\%) with severe PEP, the RAC diagnosed 319 patients (94\%) with mild, 22 patients (6\%) with moderate and no patients with severe PEP.

- Table 2 shows and compares markers of severe AP among PEP patients with a LOS 4 to 10 versus $>10$ days. Among the 132 PEP patients who had a LOS between 4 and 10 days, only $10 \%$ had markers of severe pancreatitis. On the other hand, $50 \%$ of 
- Table 2 Comparison of markers of severe AP in patients with PEP by hospital LOS $4-10$ and $>10$ days

\begin{tabular}{|l|l|l|l|}
\hline Markers of severity & LOS $\mathbf{4 - 1 0}$ days $\mathbf{( n = 1 3 2 )}$ & LOS > 10 days $(\mathbf{n}=\mathbf{1 2})$ & $\mathbf{P}$ value \\
\hline Acute fluid collection(s) & $8(6.1)$ & $1(8.3)$ & 0.8 \\
\hline Pancreatic necrosis & $2(1.5)$ & $1(8.3)$ & 0.1 \\
\hline Transient organ failure & $2(1.5)$ & $3(25)$ & $<0.001$ \\
\hline Persistent organ failure & 0 & 0 & - \\
\hline Percutaneous/surgical drainage & $1(0.8)$ & $1(8.3)$ & 0.03 \\
\hline Values are expresses as number (\%). LOS, length of stay; PEP, post-endoscopic retrograde cholangiopancreatography pancreatitis
\end{tabular}

- Table 3 Comparison of clinical indications for hospital LOS 4-10 and $>10$ days among PEP patients without markers of severe AP.

\begin{tabular}{|l|c|l|l|}
\hline & LOS 4-10 days $\mathbf{( n = 1 1 9 )}$ & LOS > 10 days (n=6) & P value \\
\hline Persistent post-procedural abdominal pain only & $56(47.1)$ & $3(50)$ & 0.24 \\
\hline Persistent nausea/vomiting and intolerance to oral intake & $14(11.8)$ & $2(33.3)$ & 0.52 \\
\hline lleus & $3(2.5)$ & 0 & 0.22 \\
\hline Fever & $11(8.4)$ & 0 & 0.25 \\
\hline Elevated liver enzymes & $13(10.9)$ & 0 & 0.28 \\
\hline Electrolyte abnormalities & $2(1.7)$ & 0 & - \\
\hline Other reasons not related to PEP & $20(16.8)$ & $1(16.7)$ & 0.15 \\
\hline Values are expresses as number (\%). LOS, length of stay; PEP, post-endoscopic retrograde cholangiopancreatography pancreatitis; AP, acutepancreatitis
\end{tabular}

patients who had a LOS $>10$ days had a clinical marker of severe AP. Of the 144 PEP patients with LOS $\geq 4$ days, only one patient (LOS 20 days) with transient respiratory failure required admission to the intensive care unit.

- Table 3 shows and compares the clinical indications for LOS $\geq 4$ versus LOS $>10$ days in PEP patients who did not have any markers of severe AP. The most common reason for an increased LOS was persistent abdominal pain, which was the only reason for a LOS between 4 and 10 days in $42 \%$ of patients and LOS $>10$ days in $25 \%$ of patients. The second most common reason for a LOS 4 to 10 days was other reasons not related to AP including urinary retention, phlebitis, comorbidities, and allergic reaction to intravenous contrast.

\section{Discussion}

Our study stratified severity of PEP by the consensus definition [3] and the RAC [6] in a large cohort of patients. We had two principal findings. First, the majority of patients that were defined as having moderate and severe PEP according to the consensus definition did not have local complications and/or organ failure. The consensus definition, therefore, overestimates the severity of pancreatitis as compared to the RAC. Secondly, LOS, a marker of severity in the consensus definition, is primarily driven by persistent post-procedural abdominal pain, nausea, vomiting and inability to tolerate oral intake. These are not clin- ical markers of severe AP; therefore, LOS is an arbitrary and highly subjective marker of severity.

In clinical practice, mild PEP is a self-limited disease that typically resolves within 1 week [6]. Nearly $10 \%$ of patients with PEP develop a severe clinical course that results in longer hospital LOS and/or additional interventions, which are associated with higher morbidity and mortality $[9,10]$. In the current study, $43 \%$ of patients were classified as having moderate or severe PEP according to the consensus definition, which is consistent with previously published studies $[2,9]$. However, $87 \%$ of these patients would be defined as having mild PEP according to the RAC. Of the $8 \%$ of patients who were classified as having severe PEP by the consensus definition, there were no cases of persistent organ failure, which is required for a diagnosis of severe AP according to the RAC. A prior systematic review of the placebo or no- stent arms of 108 PEP prophylaxis trials reported a severe PEP rate of $0.5 \%$ which is lower than the severe PEP rate of $8 \%$ in our study, both utilizing the consensus definition [2]. However, we have no severe PEP by the RAC definition as POF is extremely unusual in PEP. Based on these results, the consensus definition, as compared to the RAC, greatly overestimates the incidence of both moderate and severe PEP. Persistent post-procedural abdominal pain, nausea, vomiting and an inability to tolerate oral intake were the primary reasons for prolonged LOS for both moderate and severe PEP as defined by the consensus definition. Increased abdominal pain can be associated with peripheral and central sensitization, which is 
seen in chronic pancreatitis and functional disorders such as sphincter of Oddi dysfunction, both of which were common diagnoses among our patient cohort $[11,12]$. Pain due to air insufflation during ERCP and/or mild pancreatic inflammation mostly resolves without intervention and has little effect on outcome [13]. Symptoms of nausea and vomiting can occur for many reasons including use of drugs such as opioid analgesics, which are commonly used to treat patients with abdominal pain following ERCP [14].

PEP is the most common and costly complication of ERCP, and it is important to use a classification system that accurately defines disease severity. Although LOS is an important determinant of the cost of hospitalization, it is subject to efficiency of the health care system and has been established as an unreliable marker of disease severity across a host of medical conditions requiring hospitalization [15]. It is important to emphasize that while severe PEP is often associated with an increased LOS, this is not necessarily indicative of severe PEP, as supported by the results of our study. The optimal definition should be one that can define AP and its severity regardless of etiology. We believe that the RAC should be used for this purpose as it reflects the current and broader understanding of AP [16].

There are limitations to the current study. The primary limitation is that it is a single-center study. There may be institution-specific practices that impact LOS. However, our LOS falls within the ranges previously reported in other studies $[17,18]$. Second, our study cohort may be enriched for PEP and/or severe PEP, as our institution is a tertiary care referral center. However, the incidence of PEP at our center is similar to those reported by prior tertiary center studies. Third, we may have lost patients to follow-up who underwent ERCP but developed PEP after being discharged as well as those who were later admitted to outside hospitals with PEP. However, because our institutional protocol is to admit patients with new-onset or worsening abdominal pain after ERCP, we suspect that few patients would have been discharged only to have later developed PEP. Fourth, we may have under diagnosed PEP using the revised Atlanta classification because not all patients underwent abdominal imaging during their stay. However, these patients likely had no PEP or mild PEP since elevated serum pancreatic enzyme levels and abdominal pain are sufficient to diagnose PEP in the vast majority of patients and abdominal imaging is largely pursued in patients in whom clinicians are concerned about potential procedural complications. Fifth, there is no ICD-9 code specific for PEP. We used the combination of CPT codes for ERCP and the ICD-9 code for acute pancreatitis to identify PEP cases that were then confirmed through chart review. Sixth, we did not examine the effect of prophylaxis for PEP since nonsteroidal anti-inflammatory drugs were not utilized during the time period of our study and pancreatic stents were only employed towards the latter part of the study period as evidence supporting their use in the literature became more apparent. It is certainly possible that there was a reduction in the number of severe PEP cases due to use of prophylactic pancreatic stent insertion, but this was not the purpose of our study.

\section{Conclusion}

In conclusion, the consensus definition overestimates severity of PEP due to its overreliance on LOS. The severity of AP, rather than LOS, should be used to define severity of PEP. There is a need to either revise the current consensus definition or grade PEP severity according to the RAC.

\section{Competing interests}

Dr. Khashab is a consultant for Boston Scientific and Olympus America. Dr. Kumbhari is a consultant for Boston Scientific and Apollo Endosurgery. Dr. Kalloo is a founding member, equity holder and consultant for Apollo Endosurgery. Dr. Singh is a consultant to Abbvie, Ariel Precision Medicine and Akcea Therapeutics. Dr. Afghani is a consultant for Abbvie.

References

[1] Andriulli A, Loperfido S, Napolitano G et al. Incidence rates of postERCP complications: a systematic survey of prospective studies. Am J Gastroenterol 2007; 102: $1781-1788$

[2] Kochar B, Akshintala VS, Afghani E et al. Incidence, severity, and mortality of post-ERCP pancreatitis: a systematic review by using randomized, controlled trials. Gastrointest Endosc 2015; 81: 143 149.e9

[3] Cotton PB, Lehman G, Vennes J et al. Endoscopic sphincterotomy complications and their management: an attempt at consensus. Gastrointest Endosc 1991; 37: 383-393

[4] Akshintala VS, Hutfless SM, Colantuoni E et al. Systematic review with network meta-analysis: pharmacological prophylaxis against postERCP pancreatitis. Aliment Pharmacol Ther 2013; 38: 1325 - 1337

[5] Bradley EL 3rd. A clinically based classification system for acute pancreatitis. Summary of the International Symposium on Acute Pancreatitis, Atlanta, Ga, September 11 through 13, 1992. Arch Surg 1993; 128: $586-590$

[6] Banks PA, Bollen TL, Dervenis C et al. Classification of acute pancreatitis-2012: revision of the Atlanta classification and definitions by international consensus. Gut 2013; 62: $102-111$

[7] Vege SS, Gardner TB, Chari ST et al. Low mortality and high morbidity in severe acute pancreatitis without organ failure: a case for revising the Atlanta classification to include "moderately severe acute pancreatitis". Am J Gastroenterol 2009; 104: 710 - 715

[8] Kim E], Cho JH, Oh KY et al. The risk factors for moderately severe and severe post-endoscopic retrograde cholangiopancreatography pancreatitis according to the revised Atlanta Classification. Pancreas 2017; 46: $1208-1213$

[9] Freeman ML, Nelson DB, Sherman S et al. Complications of endoscopic biliary sphincterotomy. N Engl J Med 1996; 335: 909-918

[10] Dumonceau JM, Tringali A, Blero D et al. Biliary stenting: indications, choice of stents and results: European Society of Gastrointestinal Endoscopy (ESGE) clinical guideline. Endoscopy 2012; 44: 277-298

[11] Ceyhan GO, Bergmann F, Kadihasanoglu M et al. Pancreatic neuropathy and neuropathic pain - a comprehensive pathomorphological study of 546 cases. Gastroenterology 2009; 136: 177 -186.e1

[12] Kurucsai G, Joo I, Fejes R et al. Somatosensory hypersensitivity in the referred pain area in patients with chronic biliary pain and a sphincter of Oddi dysfunction: new aspects of an almost forgotten pathogenetic mechanism. Am J Gastroenterol 2008; 103: 2717-2725 
[13] Bretthauer M, Seip B, Aasen S et al. Carbon dioxide insufflation for more comfortable endoscopic retrograde cholangiopancreatography: a randomized, controlled, double-blind trial. Endoscopy 2007; 39: $58-64$

[14] Porreca F, Ossipov MH. Nausea and vomiting side effects with opioid analgesics during treatment of chronic pain: mechanisms, implications, and management options. Pain Med 2009; 10: 654-662

[15] Horn SD, Sharkey PD, Buckle JM et al. The relationship between severity of illness and hospital length of stay and mortality. Med Care 1991; 29: 305-317
[16] Acevedo-Piedra NG, M-H N, Rey-Riveiro M et al. New classifications of acute pancreatitis: validation of Determinant-based and Revision of the Atlanta Classification. Clin Gastroenterol Hepatol 2014; 12: 311 316

[17] Hookey LC, RioTinto R, Delhaye M et al. Risk factors for pancreatitis after pancreatic sphincterotomy: a review of 572 cases. Endoscopy 2006; 38: 670-676

[18] Ismail S, Kylanpaa L, Mustonen $\mathrm{H}$ et al. Risk factors for complications of ERCP in primary sclerosing cholangitis. Endoscopy 2012; 44: $1133-1138$ 